\title{
Diagnosis of neonatal seizure by continuous recording and rapid analysis of the electroencephalogram
}

\author{
J A EYRE, R C OOZEER, AND A R WILKINSON \\ Department of Paediatrics, John Radcliffe Maternity Hospital, Oxford
}

SUMMARY Clinical diagnosis of neonatal seizure is difficult and repeated seizures may be unrecognised. To assist in early diagnosis we recorded continuously the electroencephalogram (EEG) of very sick newborns while intensive care continued. In 25 babies at high risk of seizure a continuous record of two channels of EEG, ECG, and respiration was made for periods varying from 11 hours to 16 days. The method employed produced an EEG largely free of movement and electrical artefact which was analysed rapidly using a visual display unit. Electroencephalographic seizure activity was recorded in 20 babies, and continuous monitoring of the EEG allowed earlier recognition of seizure than was possible with clinical observation.

Seizures during the neonatal period are a considerable problem, not only because they are a symptom of neurologic or metabolic disorder, but because there is a strong association between seizure and permanent handicap in the survivors. Recent studies in animals by Wasterlain et al. suggest that the poor prognosis may not be a consequence solely of the underlying illness, but that the neonatal brain is susceptible to permanent damage as a consequence of the seizures. ${ }^{1-6}$

Watanabe, in a study of 215 newborns with clinical signs of seizure confirmed by electroencephalogram (EEG), found that $11.3 \%$ had atypical or subtle physical signs. He described the presence of subclinical seizures but did not give an incidence. ${ }^{7}$ Rose and Lombroso also found that 19 of 137 babies $(14 \%)$ with EEG evidence of seizures had very few accompanying clinical signs. ${ }^{8}$ Electroencephalographic seizure activity has been reported in newborns who were paralysed to facilitate artificial ventilation. ${ }^{910}$ The diagnosis of seizure in the newborn is therefore difficult, and repeated seizures may be unrecognised.

The reported incidence of seizures in the newborn varies from $1 \cdot 5-14 \cdot 0 / 1000$ live births. ${ }^{11-16}$ This may be the result of differences in the population at risk and in perinatal care, but it may also reflect the difficulty in making an accurate clinical diagnosis of seizure. In babies who are admitted to an intensive care nursery the incidence is as high as $3 \%{ }^{17}$

The high incidence of seizures, the difficulty in making a diagnosis, and the increasing use of paralysis to facilitate artificial ventilation indicate a need to monitor continuously the EEG of very sick babies receiving intensive care. There are major problems associated with making a prolonged record of a standard EEG. Firstly, the record is subject to artefact during the babies' spontaneous movements and during nursing and medical procedures; secondly, electrical interference poses appreciable difficulties; and finally, the recorder is large and may obstruct access to the baby. We aimed to assess a new method of making a continuous record of the EEG in very sick babies receiving intensive care and to evaluate the usefulness of these records in the diagnosis of seizure.

\section{Patients}

Twenty five babies who were considered to be at high risk of seizure because of perinatal complications were studied. The clinical details are summarised in the Table. Their birthweights ranged from 790$4160 \mathrm{~g}$, and their gestational ages at the time of the record ranged from $26-41$ weeks.

\section{Methods}

A small battery powered four channel tape recorder which measured $112 \times 86 \times 36 \mathrm{~mm}$ was used to record two channels of EEG, one channel of ECG, and on the fourth channel a time signal and an event mark or else transthoracic impedance. (Medilog 4-24 Recorder and HDX-82 Preamplifiers. Oxford Medical Systems). The tape speed allowed 24 hours 
of record to be stored on a conventional 120 minute cassette tape (TDK AD-C 120, TDK Electronic).

The EEG was recorded using silver/silver chloride cup electrodes filled with saline jelly. These were fixed to the scalp with collodion and the holes in the electrode were sealed with collodion to prevent evaporation of the conducting gel (Fig. 1). The skin/electrode impedance was measured at 6-8 hourly intervals and kept below $5 \mathrm{~K}$ ohm. When necessary the collodion seal was dissolved with acetone and additional conducting jelly was injected. The hole was then resealed with collodion. The EEG record was bipolar between $\mathrm{F}_{3}-\mathrm{P}_{3}$ and $\mathrm{F}_{4}-\mathrm{P}_{4}$ with an indifferent electrode placed on the scalp. The filter band width was set at $0 \cdot 5-100 \mathrm{~Hz}$.

The transthoracic impedance was measured using silver/silver chloride cup EEG electrodes filled with saline jelly attached to the chest with collodion and the ECG was recorded from silver/silver chloride disposable electrodes.

The tape record was reviewed at intervals determined by the clinical condition of the baby and by the previous findings. The tape was replayed on a visual display unit (PMD 12 Virgo Special, Oxford Medical Systems) that automatically presented the

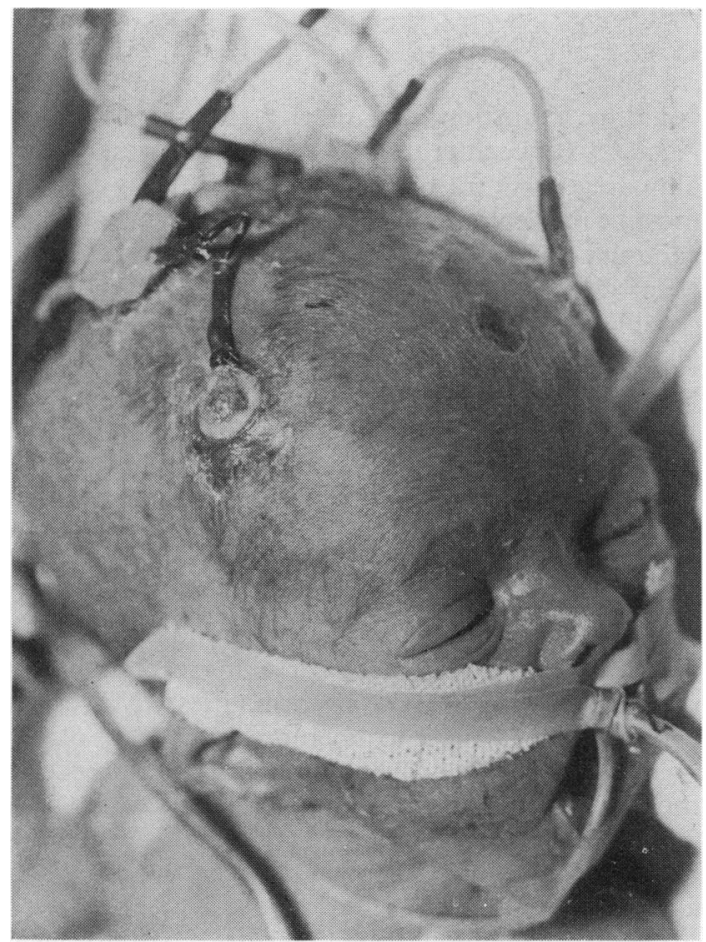

Fig. 1 Attachment of electrodes and preamplifiers to the scalp wjth collodion. recorded signal as 'pages' of data comprising 8 or 16 seconds of EEG, ECG, and thoracic impedance record. The 'pages' were presented at a 20th or a 60 th of the real time; in the latter case allowing rapid visual scanning of 24 hours of data in 24 minutes. Sections of the record showing abnormality could then be reviewed at a manually operated speed. During the recording, a bedside monitor (Oxford Medical Systems) was used to display simultaneously the EEG, the ECG, the heart rate, the chest wall movement and, in those babies with intra-arterial catheters, the blood pressure wave form.

A standard definition of an electroencephalographic seizure discharge was used. This was an orderly build up and termination of rhythmic sharp and slow waves, where the discharge persisted for longer than 20 seconds (Fig. 2), or the sudden onset and termination of repetitive and rhythmical waves of a single frequency in the 4-13 Hz range (Fig. 3) when the discharge persisted for longer than 20 seconds. ${ }^{18}$

In a preliminary study in which a standard EEG recorder was used, we found that if the electrodes alone were fixed to the skin then artefact resulting from movement was an appreciable problem. This

Boy Age 2days ( 26 weeks gestation)

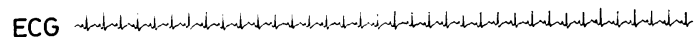
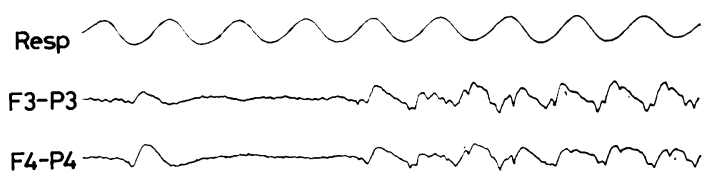

$100 \mu \mathrm{V}$ L

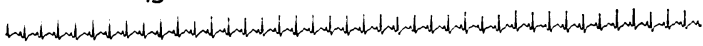

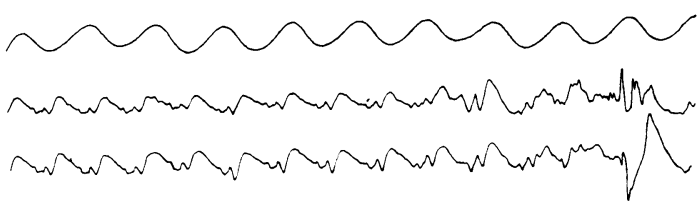

إl

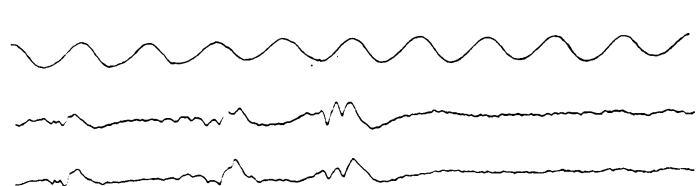

Fig. 2 Siezure discharge with abrupt onset of synchronous and symmetrical rhythmic sharp and slow waves in the right and left channel of the EEG. 
Girl Age 6 weeks ( 24 weeks gestation)
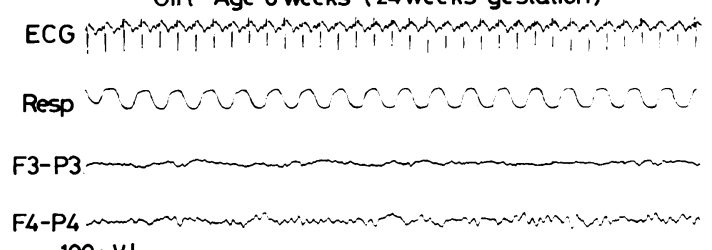
$100 \mu \mathrm{VVL}$

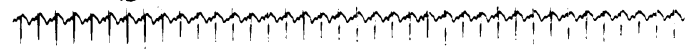

aravaruaranarara

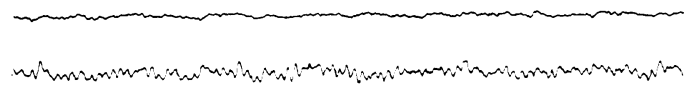

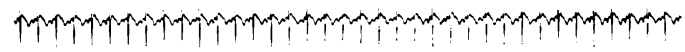

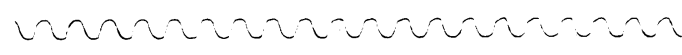

Fig. 3 Seizure discharge of rhythmical waves in the $\theta$ frequency in the right channel of the EEG.

artefact occurred in both the EEG and the transthoracic impedance record, and it obscured long sections of the record (Fig. 4). To reduce movement artefact in the EEG record the electrodes were placed at the $\mathrm{F}_{3}-\mathrm{P}_{3}$ and $\mathrm{F}_{4}-\mathrm{P}_{4}$ positions where they would not be in contact with the mattress. To protect the electrodes from displacement during movement, the miniaturised preamplifiers $(13 \times$ $13 \times 6 \mathrm{~mm}$ ) were also fixed to the scalp with collodion. Thus displacement during movement occurred at the scalp/preamplifier junction and not at the EEG electrode (Fig. 1). To minimise displacement of the electrodes used to record transthoracic impedance, the patient leads were fixed to the chest wall using adhesive tape and collodion. During application of the electrodes the clinical condition of the baby, the heart rate, and the blood pressure were carefully observed for adverse changes.

\section{Results}

The application of the electrodes took approximately 30 minutes and did not disturb the clinical condition of any of the babies. Recording did not interfere with intensive care; nursing and medical procedures could continue; and the tape recorder remained connected during all investigations and

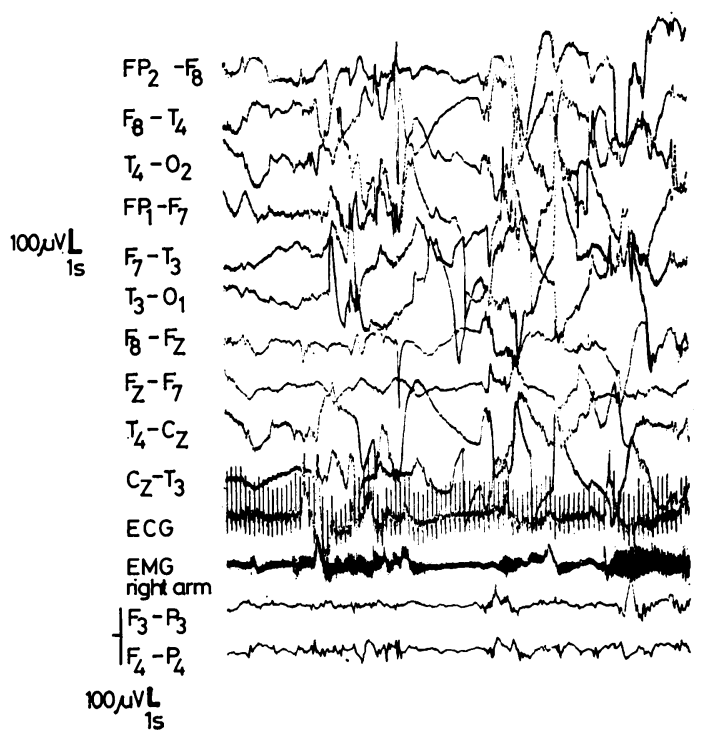

Fig. 4 Movement artefact in a standard 9 channel $E E G$ record compared with an artefact free simultaneously recorded 2 channel EEG from electrodes and preamplifiers attached to the scalp with collodion.

could be transported with the baby if necessary. Access to the anterior fontanelle was preserved to allow regular ultrasound scanning of the brain, and most of the scalp remained available for intravenous cannulae (Fig. 1). Only after 5-6 days did the electrodes need refixing to the scalp. The only complication from the procedure was a localised allergic skin reaction to collodion in one baby; this subsequently resolved completely.

The recording period varied between 11 hours-16 days; the median was 72 hours and a total time was 2680 hours (Table). During prolonged records approximately 30 minutes of attention per day was necessary to maintain a satisfactory skin/electrode impedance. The time taken to analyse a 24 hour record varied from 30 minutes when no abnormality was found to three hours for a more complicated record showing EEG abnormalities. Movement artefact was reduced to no more than $10 \%$ of any one tape, and there was no electrical interference on the records, despite the complex electrical environment in the intensive care nursery-presumably because of the short distance between the electrodes and the preamplifier.

Electroencephalographic seizure activity was recorded in 20 of 25 babies studied (see Table). In 11 babies clinical signs accompanied some but 
not all of the seizures. Four babies were paralysed during the seizure activity, and in five there were no associated clinical signs. For 14 babies the seizure activity was confined to paroxysmal discharges of rhythmic sharp or slow waves (Fig. 2), in five both paroxysms of rhythmic sharp or slow waves and paroxysmal discharges of rhythmical activity in the $\alpha$ or $\theta$ frequency were found (Fig. 3), and in one baby the seizure activity consisted solely of paroxysmal discharges of rhythmical activity in the $\alpha$ or $\theta$ frequency. The electroencephalographic seizure activity seen in the babies without clinical signs of seizure was not different from that seen in babies with accompanying signs.

A total of 3619 episodes of electroencephalographic seizure activity were recorded (Table); 2279 $(63 \%)$ occurred synchronously and symmetrically in both the right and left channels, $108(3 \%)$ occurred simultaneously but asynchronously in the right and left channel, while $1232(34 \%)$ were recorded in only the right or left channel. Eighty two per cent of the seizure discharges comprised paroxysms of rhythmical sharp and slow waves, and $18 \%$ of paroxysms of rhythmical activity in the $\alpha$ or $\theta$ frequencies. Metabolic abnormalities accompanied the perinatal complications and seizures in 15 babies, but electroencephalographic seizures continued despite correction of these disorders. Seizure activity stopped within 6 hours of giving anticonvulsant drugs in only 4 babies, although serum values were within the accepted therapeutic range in all babies.

\section{Discussion}

The pathogenesis of brain damage as a consequence of repeated seizures has been attributed to neuronal necrosis secondary to increased neuronal energy requirement in the presence of the hypoxaemia that may accompany the motor activity of a seizure..$^{19}$ In his studies in animals, however, Wasterlain found that seizures during the neonatal period (induced by indirect electroconvulsive shock, flurothyl, bicuculline, or fever) inhibit brain DNA synthesis, permanently reduce the number of brain cells and the brain size, and delay behavioural milestones even in animals paralysed and adequately ventilated and oxygenated throughout the seizure. Similar effects have not been found in studies of mature animals. ${ }^{1-6}$

These studies in animals suggest that the newborn may be particularly susceptible to adverse neurological consequences from repeated uncontrolled seizures. The American National collaborative perinatal project ${ }^{20}$ found that cerebral palsy and mental retardation were strongly related to the duration of the longest seizure and the number of days in which repeated seizure occurred in the neonatal period. Plum, Posner, and Troy, ${ }^{21}$ in studies of dogs and primates, reported a loss of cerebral blood flow autoregulation, a rise in mean arterial blood pressure, and a consequent rise of $200 \%$ in cerebral blood flow during seizures. Lou and Friis-Hansen ${ }^{22}$ reported an increase in the mean arterial blood pressure of up to $40 \mathrm{~mm} \mathrm{Hg}$, and Perlman and Volpe ${ }^{23}$ found increased cerebral blood flow velocity in the neonates during seizures with minimal motor activity. In view of the possible disturbances of autoregulation of cerebral blood flow in ill newborns, an abrupt increase in cerebral blood flow and intracranial pressure may accompany seizures and contribute to the pathogenesis of intraventricular haemorrhage. ${ }^{24}$ These data also suggest that prompt control of electroencephalographic seizure activity may improve prognosis.

A review of the published reports on neonatal convulsions shows that most neonatal seizures occur within three days after birth, and that the percentage of seizures during this time varies from $43 \%$ as found by Brown, Cockburn, and Forfar to $86 \%$ as found by Burke. ${ }^{1215162526}$ A continuous record of the EEG in ill babies during the first three days after birth will therefore identify most babies who have neonatal seizures.

The continuous record of the EEG enabled quantification of both the number of episodes and the total duration of electroencephalographic seizure activity during an acute neurological illness. With this method it is possible, for the first time, to relate the total duration of electroencephalographic seizure activity and abnormal interictal activity to the eventual outcome.

The continous record also allows an assessment of the change in frequency of seizure activity over time and in response to treatment. While the aim of this study was not to assess the efficacy of treatment of seizure disorders, the unexpectedly high numbers of seizures, despite apparently adequate serum values of anticonvulsant drugs, suggests that antiseizure treatment did not alter the frequency of seizure activity in these babies.

The method of continuously recording the EEG does have limitations. Without the constant presence of a technician the recognition of the clinical signs of seizure will depend on intermittent nursing and medical observations, and subtle signs may be overlooked. Two channels of EEG may not show all focal discharges and the localisation of their origin will continue to require a standard 12-16 channel EEG. These disadvantages are balanced by a reduction in disturbance to the baby and minimal interference with nursing care. In addition, access is 
maintained to the anterior fontanelle for ultrasound studies and to scalp vessels for venous or arterial cannulation.

The ability to diagnose seizure in those babies who are paralysed and in those with minimal motor signs of seizure depends upon the electroencephalographic finding of seizure activity. We describe a practical non-invasive method to monitor continuously the EEG in sick newborn babies.

We thank Sir Peter Tizard for advice, Drs P Brankin and J Morris of Oxford Medical Systems for technical assistance, the medical and nursing staff for their cooperation, Mrs A Smith for advice, and Miss S Ruddock who typed the manuscript. The study was supported by Action Research for the Crippled Child.

\section{References}

1 Wasterlain CG. Breakdown of brain polysomes in status epilepticus. Brain Res 1972;39:278-84.

2 Wasterlain CG, Plum F. Vulnerability of developing rat brain to electroconvulsive seizures. Arch Neurol 1973;29: 38-45.

3 Wasterlain CG. Inhibition of cerebral protein synthesis by epileptic seizures without motor manifestations. Neurology (Minneap) 1974;24:175-80.

4 Wasterlain CG. Mortality and morbidity from serial seizures. An experimental study. Epilepsia 1974;15: 155-76.

5 Wasterlain CG. Effects of neonatal status epilepticus on rat brain development. Neurology (Minneap) 1976;26: 975-86.

6 Wasterlain CG. Does anoxemia play a role in the effects of neonatal seizures on brain growth? An experimental study in the rat. EurNeurol 1979;18:222-9.

7 Watanabe K, Hara K, Miyazaki S, et al. Electroclinical studies of seizures in the newborn. Folia Psychiatr Neurol Jpn 1977;31:383-92.

8 Rose AL, Lombroso CT. Neonatal seizure states. Pediatrics 1970;45:404-25.

9 Staudt F, Roth JG, Engel RC. The usefulness of electroencephalography in curarized newborns. Electroencephalogr Clin Neurophysiol $1981 ; 51: 205-8$.

10 Eyre JA, Oozeer RC, Wilkinson AR. Continuous electroencephalographic recording to detect seizures in paralysed newborns. Br Med J 1983;286:1017-8.

11 Eriksson M, Zetterstrom R. Neonatal convulsions.
Incidence and causes in the Stockholm area. Acta Paediatr Scand 1979;68:807-11.

12 Burke JB. The prognostic significance of neonatal convulsions. Arch Dis Child 1954;29:342-5.

13 Craig WS. Convulsive movements occurring in the first 10 days of life. Arch Dis Child 1960;35:336-44.

14 Keen JH, Lee D. Sequelae of neonatal convulsions. Arch Dis Child 1973;48:542-6.

15 Dennis J. Neonatal convulsions: aetiology, late neonatal status and long-term outcome. Dev Med Child Neurol $1978 ; 20: 143-58$.

16 Brown JK, Cockburn F, Forfar JO. Clinical and chemical correlates in convulsions of the newborn. Lancet 1972;i: 135-9.

17 Ment LR, Freedman RM, Ehrenkranz RA. Neonates with seizures attributable to perinatal complications. Am J Dis Child 1982;136:548-50.

18 Fenichel GM. Neonatal electroencephalography: neonatal neurology. London and Edinburgh: Churchill Livingstone, 1980:235-9.

19 Meldrum BS, Brierley JB. Neuronal loss and gliosis in the hippocampus following repetitive epileptic seizures induced in adolescent baboons by allylglycine. Brain Res 1972;48:361-5.

20 Mellits ED, Holden KR, Freeman JM. Neonatal seizures II. A multivariate analysis of factors associated with outcome. Pediatrics 1982;70:177-85.

21 Plum F, Posner JB, Troy B. Cerebral metabolic and circulatory responses to induced convulsions in animals. Arch Neurol $1968 ; 18: 1-13$.

${ }^{22}$ Lou HC, Friis-Hansen B. Arterial blood pressure elevations during motor activity and epileptic seizures in the newborn. Acta Paediatr Scand 1979;68:803-6.

${ }^{23}$ Perlman JM, Volpe JJ. The effects of seizures on cerebral blood flow velocity intracranial pressure and systemic blood pressure in the preterm infant (Abstract). Pediatr Res 1982;16:339A.

24 Lou HC, Lassen NA, Friis-Hansen B. Impaired autoregulation of cerebral blood flow in the distressed newborn infant. J Pediatr 1979;94:118-21.

25 Harris R, Tizard JPM. The electroencephalogram in neonatal convulsions. JPediatr 1960;57:501-20.

${ }^{26}$ Knauss TA, Marshall RE. Seizures in a neonatal intensive care unit. Dev Med Child Neurol 1977;19:719-28.

Correspondence to $\operatorname{Dr} A \mathbf{R}$ Wilkinson, Neonatal Unit, Department of Paediatrics, John Radcliffe Maternity Hospital, Headington, Oxford OX3 9DU.

Received 22 June 1983 